\title{
Effect of Modified Solvent Casting/Particulate Leaching (SCPL) Technique on the Properties of Bioactive Glass Reinforced Polyurethane Scaffold for Biomedical Applications
}

\author{
Ong Chia Chia, Intan Syazana Suhaimin, Syamimi Abu Kassim, \\ Syazana Ahmad Zubir and Tuti Katrina Abdullah*
}

School of Materials and Minerals Resources Engineering, Universiti Sains Malaysia, Engineering Campus, 14300 Nibong Tebal, Pulau Pinang, Malaysia

*Corresponding author: tutikatrina@usm.my

Published online: 25 December 2019

To cite this article: Chia, O. C. et al. (2019). Effect of modified solvent casting/ particulate leaching (SCPL) technique on the properties of bioactive glass reinforced polyurethane scaffold for biomedical applications. J. Phys. Sci., 30(Supp. 2), 115-126, https://doi.org/10.21315/jps2019.30.s2.10

To link to this article: https://doi.org/10.21315/jps2019.30.s2.10

\begin{abstract}
Salt leaching technique is commonly used in the preparation of porous scaffolds for tissue engineering due to easy control of pore structure. However, distribution of the salt particles within polymer solution using this technique is difficult to be controlled and causes a problem of residual salt in scaffolds. Solvent casting technique is often combined with salt leaching technique to produce porous scaffold. In this present work, modification of solvent casting/particulate leaching (SCPL) technique was developed which involved a conventional SCPL method combined with a step of centrifugation using different centrifugal speeds of $1500 \mathrm{rpm}, 2000 \mathrm{rpm}, 2500 \mathrm{rpm}$ and $3000 \mathrm{rpm}$ to produce $45 S 5$ bioactive glass $(B G)$ reinforced polyurethane (PU) scaffolds. PU-BG scaffolds were fabricated with mass ratio of $90 P U: 10 B G$ and with 52 wt\% hard segment content of PU. Sodium chloride ( $\mathrm{NaCl}$ ) and sodium bicarbonate $\left(\mathrm{NaHCO}_{3}\right)$ were used as the leaching agent to create pores in the scaffolds. The physical, structural, morphological and mechanical properties of scaffolds were studied by Fourier transform infrared (FTIR) spectroscopy, scanning electron microscopy (SEM) equipped with energy dispersive X-ray spectroscopy (EDX), porosity test and compression test. Highly porous scaffolds with good interconnectivity were obtained by enhanced SCPL method with porosity ranging from $88 \%$ to $90 \%$. The porosity increased with an increase in the centrifugal speed up to $2500 \mathrm{rpm}$. While the mechanical properties of the scaffolds were compromised as lower compressive strength ranging from $0.17 \mathrm{Mpa}$ to $0.66 \mathrm{MPa}$ were obtained by modified SCPL method as compared to $0.90 \mathrm{MPa}$ by conventional SCPL method.
\end{abstract}

Keywords: Scaffold, bioactive glass, polyurethane, solvent casting/particulate leaching, SCPL 


\section{INTRODUCTION}

Nowadays, current advances are being made with the developments of tissueengineered products. Scaffold-based tissue engineering consists of an artificial extracellular matrix (ECM) and three-dimensional (3D) scaffolds containing living cells and regulating biomolecules. ${ }^{1}$ The scaffold mimics the central component that imitate ECM, whereas the cells and growth factors will interact with ECM to regenerate targeted tissues. ${ }^{2}$ Mechanical properties of scaffold should match and bond to host bone without the scar tissue formation, besides providing a sufficient support for new bone formation. A porous and interconnected scaffold structure are important to ensure the waste products can be easily diffused out. The products of scaffold degradation should be able to exit the body without any reaction with other organs and the surrounding tissues. ${ }^{1}$

To fabricate such tissue scaffolds, many different fabrication techniques have been developed and are classified into two types, which are conventional and advanced methods. Conventional techniques include solvent-casting, particulate-leaching, and freeze drying which enable the fabrication of scaffolds with interconnected porous structures. Solvent casting/particulate leaching (SCPL) method is the most commonly used technique due to its simple operation. ${ }^{3}$ Introducing a combination of materials during scaffold manufacture to fabricate composite scaffold has enhanced greater bioactivity and increased the chance of achieving structural biomimicry. ${ }^{4}$ Polyurethanes (PU) are widely used in medical field as wound dressings, vascular grafts, artificial heart valves and also tissue scaffolds. $\mathrm{PU}$ is considered as one of the biopolymers for bone tissue engineering due to its excellent biocompatibility. Bioceramics such as bioactive glass (BG) are capable to react with the physiological environment by creating a strong bonding interface made up of a bone-like hydroxyapatite layer, resulting in a stable fixation of the material to the host tissue. ${ }^{5}$

According to Zhu and Chen, highly porous scaffolds with porosity reaches $93 \%$ and average pore sizes up to $500 \mu \mathrm{m}$ can be obtained by using SCPL technique. ${ }^{6}$ However, the distribution of the salt particles within the polymer solution using this technique is often not uniform due to the difference in density between the liquid polymer solution and the solid salt. The degree of salt particles in direct contact with each other are not well controlled. This technique may introduce residual salt problem in scaffolds which will lead to poor pore interconnectivity. To solve this problem, an enhanced SCPL method which involves the use of centrifugation technique was proposed to improve the pore interconnectivity of the scaffolds. The main objective of this study is to investigate the properties of BG reinforced PU scaffold fabricated by SCPL which involves the centrifugation 
method. Improvement in pore interconnectivity may lead to effective use of porous biodegradable scaffolds in different tissue engineering strategies, especially in neural and muscular applications.

\section{EXPERIMENTAL}

\subsection{Synthesis of Bioglass}

Bioglass $45 \mathrm{~S} 5$ with $45.0 \mathrm{wt} \%$ silicon dioxide $\left(\mathrm{SiO}_{2}\right), 24.5 \mathrm{wt} \%$ sodium oxide $\left(\mathrm{Na}_{2} \mathrm{O}\right), 24.5 \mathrm{wt} \%$ calcium oxide $(\mathrm{CaO})$ and $6 \mathrm{wt} \%$ phosphorus pentoxide $\left(\mathrm{P}_{2} \mathrm{O}_{5}\right)$ were synthesised by melt quenched technique to produce BG. The powders were melted in Lenton Tube EHF 1800 furnace at $1400^{\circ} \mathrm{C}$ with heating rate of $10^{\circ} \mathrm{C} \mathrm{min}{ }^{-1}$ followed by soaking for $1 \mathrm{~h}$. The glass melt was immediately waterquenched into deionised water after the heating completed. The fabricated glasses were then crushed using agate mortar and ball milled at $400 \mathrm{rpm}$ for $1 \mathrm{~h}$ with ballto-powder ratio of 10:1 to mill BG into powder form.

\subsection{Synthesis of PU}

PU (PEARLTHANE 11T80) was synthesised by two step-polymerisation technique. First, polycaprolactone diol (PCL) was put in oven overnight at $80^{\circ} \mathrm{C}$ to melt into liquid form, then mixed with 4,4'-methylenebis (cyclohexyl isocyanate) (HMDI) and stirred again for $2.5 \mathrm{~h}$ at $80^{\circ} \mathrm{C}$ under the flow of nitrogen gas. After sintering completed, the pre-polymer products were formed. Second, butanediol (BD) which is the chain extender was added to the polymer followed by the addition of two drops dibutyltin dilaurate (DBTDL) catalyst. The mixture was mixed at $50 \mathrm{rpm}$ using HAAKE internal mixer for $15 \mathrm{~min}$ at $90^{\circ} \mathrm{C}$. The molar ratio used for PCL:HMDI:BD was 1:8:7 which having hard segment (HS) content of $52 \mathrm{wt} \%$ based on Equation 1: ${ }^{7}$

$$
\mathrm{HS} \%=x+\frac{n M_{H M D I}+n M_{B D}}{n M_{P C L} n M_{H M D I}+n M_{B D}}
$$

\subsection{Fabrication of PU-BG Scaffold Composites}

The PU-BG scaffold was fabricated by SCPL technique. PU was dissolved in tetrahydrofuran (THF) (Sigma-Aldrich) with concentration of $19 \mathrm{wt} / \mathrm{vol} \%$ at $50^{\circ} \mathrm{C}$ and stirred for $2 \mathrm{~h}$ to form PU solution. Then, $10 \mathrm{wt} \%$ of BG powder was added into $\mathrm{PU}$ solution and stirring was continued for $1.5 \mathrm{~h}$ at $50^{\circ} \mathrm{C}$ to allow homogenisation of BG dispersion. Leaching agents of $65 \mathrm{wt} \%$ sodium chloride $(\mathrm{NaCl})$ and $35 \mathrm{wt} \%$ sodium bicarbonate $\left(\mathrm{NaHCO}_{3}\right)(\mathrm{MERCK}$ Milipore) were added into the mixture 
with mass ratio of PU:leaching agent at 1:6 and stirred to achieve homogeneous solution. Different stirring methods which included conventional SCPL and enhanced SCPL (combination of centrifugation with conventional SCPL) methods were applied and shown in Table 1. The homogeneous solution was transferred into Teflon mould and dried in fume cupboard for three days at room temperature to allow evaporation of THF. The samples were then immersed in deionised (DI) water to leach out the leaching agents. The DI water was changed every $6 \mathrm{~h}$ for seven days. Lastly, the scaffold was dried overnight in an oven at $80^{\circ} \mathrm{C}$.

Table 1: Stirring methods and speed used in fabrication of scaffolds.

\begin{tabular}{lcc}
\hline Stirring method & Speed (rpm) & Time (min) \\
\hline Conventional SCPL $^{8}$ & 1000 & 60 \\
& 1500 & 10 \\
Enhanced SCPL (combination with centrifugation) & 2000 & 10 \\
& 2500 & 10 \\
& 3000 & 10 \\
\hline
\end{tabular}

\subsection{Characterisation}

FTI was performed to identify the functional groups $(\mathrm{C}=\mathrm{O}, \mathrm{C}-\mathrm{N}$, and $\mathrm{Si}-\mathrm{O})$ present within a sample. PU samples were heated from room temperature to $800^{\circ} \mathrm{C}$ with a heating rate of $10^{\circ} \mathrm{C} \mathrm{min}-1$ under the flow of nitrogen gas. Field emission scanning electron microscope (FESEM) equipped with energy dispersive X-ray spectroscopy (EDX) was used to characterise the pore structure and elemental composition of the scaffold. Compressive strength of the PU-BG scaffolds was determined using Instron 5982 Universal Testing Machine (UTM) with a load of $5 \mathrm{kN}$ and a crosshead speed of $1 \mathrm{~mm} \mathrm{~min}^{-1}$. The maximum compressive load and compressive modulus of scaffold samples were determined at 50\% compressive strain. The test was carried out at ambient temperature.

\subsection{Calculation of Total Porosity}

Total porosity (\%) of scaffolds was calculated using Equation 2, where $\rho_{\text {scaffold }}$ is the bulk density of the scaffold sample, $\rho_{\text {material }}$ is the true density of the composite material in solid phase. ${ }^{9}$

$$
\% \text { Porosity }=\left[1-\frac{\rho_{\text {scaffold }}}{\rho_{\text {material }}}\right] \times 100 \%
$$


The density of BG and PU were measured by densitometer (Presica XB 220A). The true density, $\rho_{\text {polymer }}$ of the material was measured using the Rule of Mixture formula in Equation 3, where $\rho_{P U-B G}$ is the density of scaffold, $\rho_{P U}$ is the density of $\mathrm{PU}, \rho_{B G}$ is the density of BG, $X_{P U}$ is the volume fraction of PU in PU-BG scaffold and $X_{B G}$ is the volume fraction of BG in PU-BG scaffold. ${ }^{9}$

$$
\rho_{P U-B G}=\rho_{P U}\left(X_{P U}\right)+\rho_{B G}\left(X_{B G}\right)
$$

Subsequently, bulk density of the scaffold was calculated using Equation $4 .{ }^{9}$

$$
\rho_{\text {scaffild }}=\frac{\text { mass }}{\text { volume }}
$$

\section{RESULTS AND DISCUSSION}

\subsection{Functional Group Identification}

Figure 1 shows the FTIR spectra for PU, BG powder and PU-BG scaffold. The FTIR spectra for PU, shown in Figure 1(a), shows the spectrum peak at $3333 \mathrm{~cm}^{-1}$ corresponding to $\mathrm{N}-\mathrm{H}$ stretching vibration which was characteristic bands for urethane. The peak appeared at $1725 \mathrm{~cm}^{-1}$ corresponded to carbonyl $(\mathrm{C}=\mathrm{O})$ group while the peak at $1693 \mathrm{~cm}^{-1}$ was due to hydrogen bonded carbonyl, amide I $(\mathrm{C}=\mathrm{O})$ stretching. The sharp peaks at $2925 \mathrm{~cm}^{-1}$ and $2858 \mathrm{~cm}^{-1}$ are associated with $-\mathrm{CH}_{2}$ asymmetric and symmetric stretching. No peaks belonging to $\mathrm{NCO}$ bond exist in Figure 1(a), indicating the complete reaction between isocyanate and hydroxyl to form urethane bond. FTIR reflectance spectra of BG, shown in Figure 1(b) exhibited vibrational bands at around $500 \mathrm{~cm}^{-1}$ which was due to Si$\mathrm{O}-\mathrm{Si}$ bending. Subsequently, the presence of characteristic peak at $935 \mathrm{~cm}^{-1}$ was due to Si-O stretching and $1027 \mathrm{~cm}^{-1}$ was due to Si-O-Si stretching (asymmetric). FTIR spectra of the scaffold fabricated using conventional SCPL method as in Figure (1c) show Si-O stretching and asymmetric Si-O-Si stretching bands were located at $982 \mathrm{~cm}^{-1}$ and $1045 \mathrm{~cm}^{-1}$. There are no significant differences in trend observed between scaffolds produced by conventional and enhanced SCPL method (centrifuge at $1500 \mathrm{rpm}, 2000 \mathrm{rpm}, 2500 \mathrm{rpm}$ and $3000 \mathrm{rpm}$ ). As the compositions used ( $90 \mathrm{wt} \%$ of PU and $10 \mathrm{wt} \%$ of BG) are the same for all scaffolds, FTIR spectra show no significant difference between all scaffolds. 
(a)

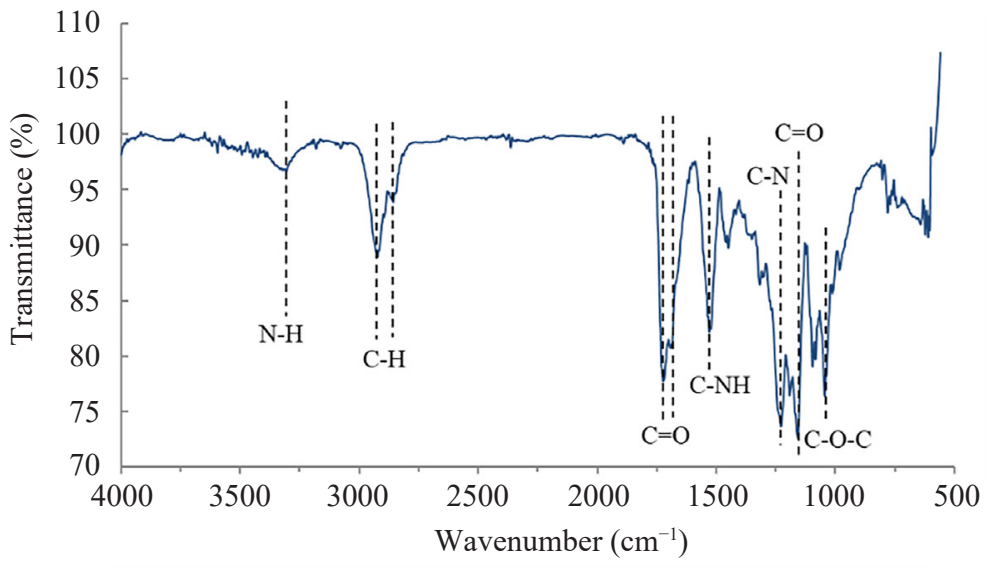

(b)

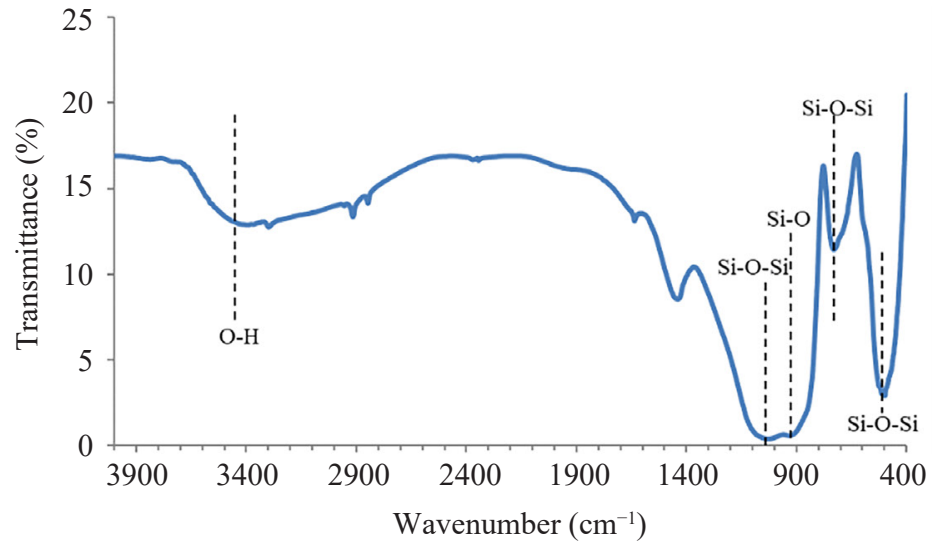

(c)

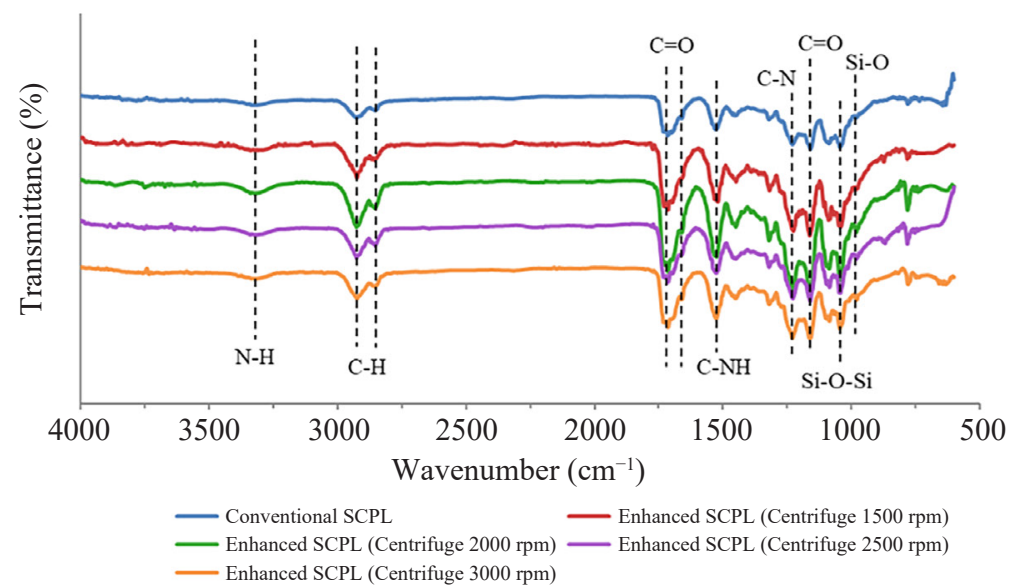

Figure 1: FTIR spectra of (a) PU, (b) BG powder, and (c) scaffold fabricated using conventional SCPL and enhanced SCPL methods. 


\subsection{Surface Morphology and Elemental Distribution of Scaffold}

Figure 2 shows the morphology of scaffolds fabricated using conventional SCPL and enhanced SCPL (centrifugation at 1500, 2000, 2500 and $3000 \mathrm{rpm}$ ) methods. Enhanced SCPL method at all centrifugal speed shows a higher porosity which contains micropores and macropores structure relative to the conventional SCPL method. Enhanced SCPL method reveals the pore interconnectivity of scaffold has increased as compared to the conventional SCPL as centrifugal force enabled the particles to be densely packed and having a direct contact with each other. ${ }^{3}$ There is no significant difference observed among the scaffolds fabricated by enhanced SCPL method regardless of stirring speed used. Both methods show that BG particles which appeared as white dots were well-dispersed and homogeneously embedded in the walls of PU matrix.

EDX anaysis estimates that the amount of $\mathrm{Na}$ presents in the scaffolds fabricated by conventional SCPL was $2.95 \mathrm{wt} \%$ shown in Figure 3(a). For enhanced SCPL with the centrifugal speed of at $1500 \mathrm{rpm}, 2000 \mathrm{rpm}, 2500 \mathrm{rpm}$ and $3000 \mathrm{rpm}$, the amount of Na present in scaffolds were $1.92 \mathrm{wt} \%, 1.6 \mathrm{wt} \%, 1.9 \mathrm{wt} \%$ and $2.91 \mathrm{wt} \%$, respectively, shown in Figures 3(b-e). The lower amount of $\mathrm{Na}$ traced in all scaffolds suggests that the leaching agents have been leached out as interconnected pores allow the salts to be removed easily. Interconnected pores are essential to ensure that no residual salt are left inside the scaffolds. The pore size of the scaffolds appears to be dependent on the size of leaching agents: $\mathrm{NaCl}$ $(100-380 \mu \mathrm{m})$ and $\mathrm{NaHCO}_{3}(65-280 \mu \mathrm{m})$.

\subsection{Total Porosity}

The bulk density, true density and the total porossity of the scaffolds are tabulated in Table 2. Total porosity increased with an increase in the centrifugal speed up to $2500 \mathrm{rpm}$, i.e., $88.55 \%$ (1500 rpm), 89.00\% (2000 rpm) and 90.42\% (2500 rpm). However, the total porosity decreased to $87.93 \%$ when the centrifugal speed was increased to $3000 \mathrm{rpm}$. When centrifugal force is applied, the salt particles are being pushed to the bottom of the tubes at a rate proportional to the force applied. At the same time, polymer solution will fill the gap among the packed salt particles. ${ }^{3}$ As the centrifugal speed increases, centrifugation force will be increased. Hence, the viscosity of the polymer solution can be overcome easily when centrifugal speed increased from $1500 \mathrm{rpm}$ to $2500 \mathrm{rpm}$, enabling more salt particles to be densely packed and leading to more porous and interconnected structures. At $3000 \mathrm{rpm}$, the high centrifugal speed resulted in the higher salt concentration at the bottom of the tube, thereby causing a difficulty for the polymer solution to enter the gaps between the salt particles. As a result, the total porosity shows a slight decrement at $3000 \mathrm{rpm}$ of centrifugal speed. 
(a)

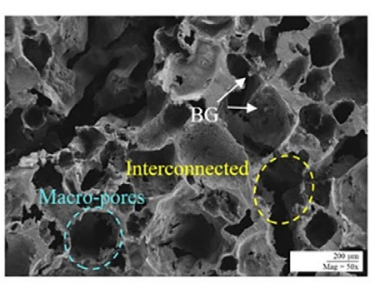

(d)

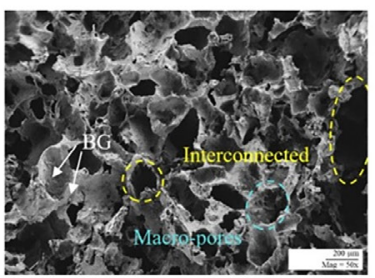

(g)

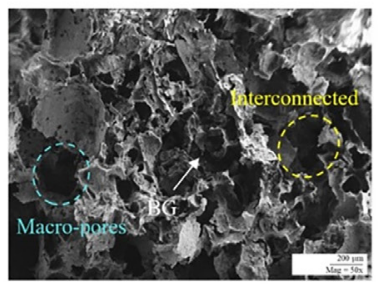

(j)

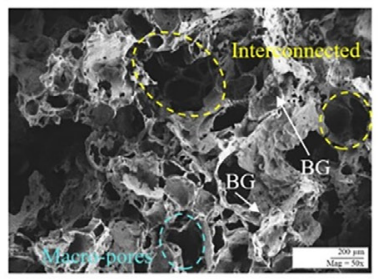

$(\mathrm{m})$

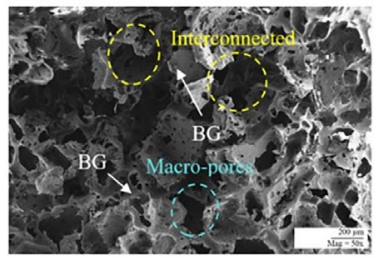

(b)

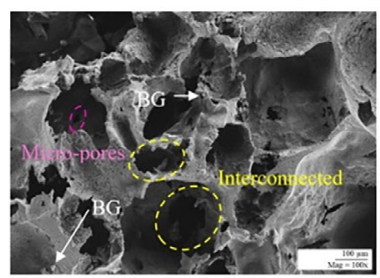

(e)

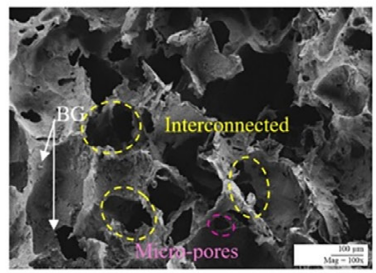

(h)

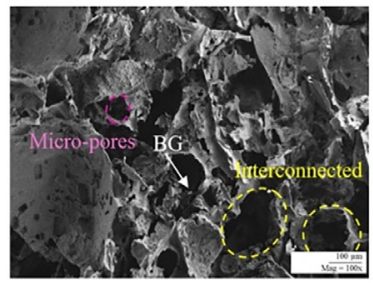

(k)

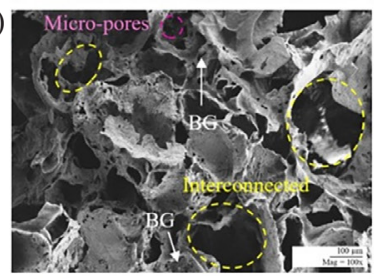

(n)

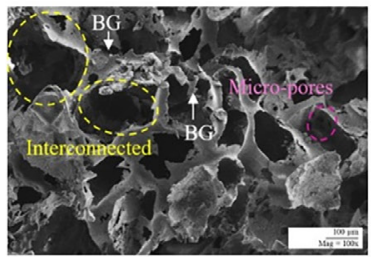

(c).

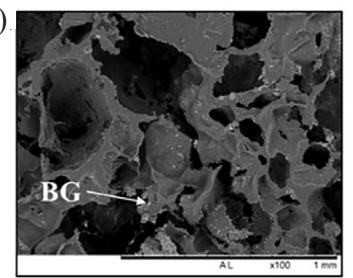

(f)

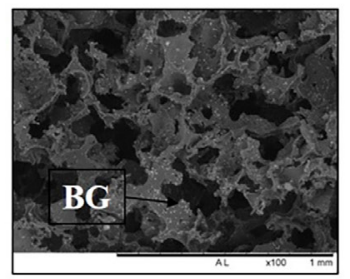

(i)

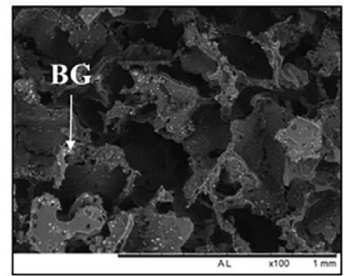

(1)

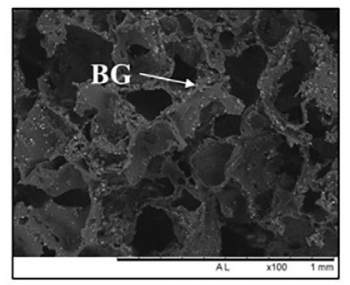

(o)

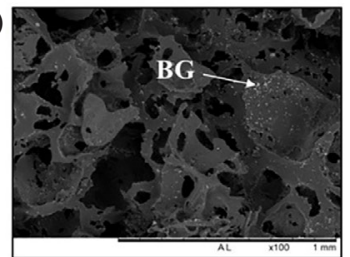

Figure 2: Micrograph of the porous structure for $(a-c)$ conventional SCPL, $(d-f)$ enhanced SCPL with centrifugal speed of $1500 \mathrm{rpm},(\mathrm{g}-\mathrm{i}) 2000 \mathrm{rpm},(\mathrm{j}-1) 2500 \mathrm{rpm}$, and (m-o) $3000 \mathrm{rpm}$. 


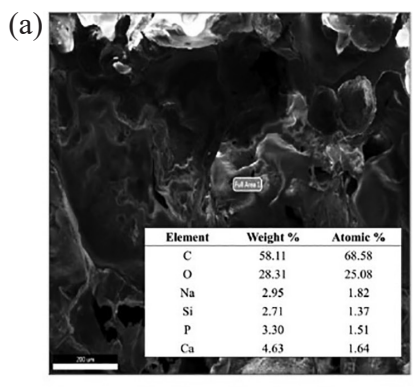

(d)

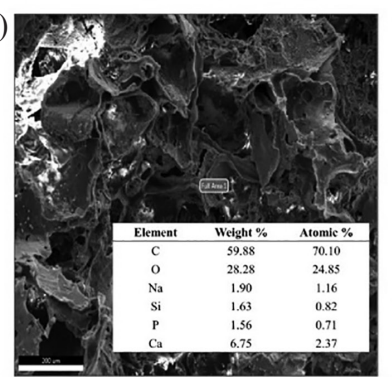

(b)
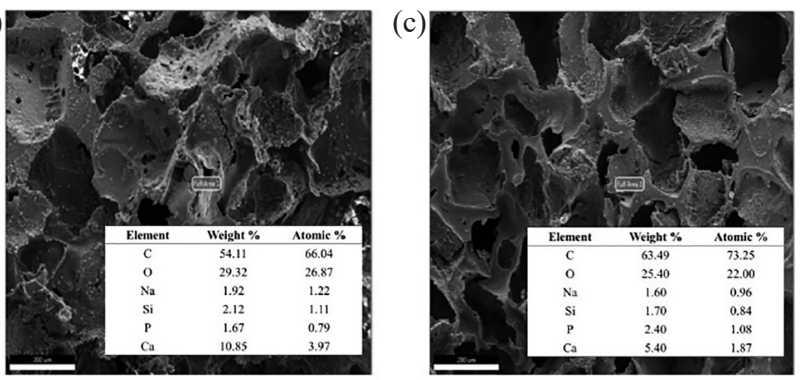

(e)

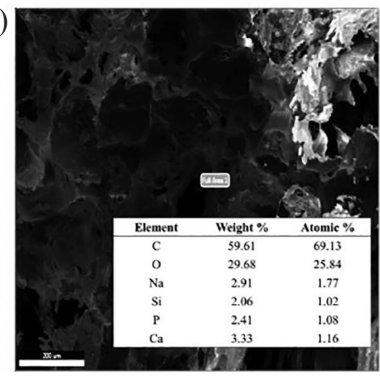

Figure 3: EDX results of scaffold fabricated by (a) conventional SCPL; and enchanced SCPL which centrifuge at (b) $1500 \mathrm{rpm}$, (c) $2000 \mathrm{rpm}$, (d) $2500 \mathrm{rpm}$, and (e) $3000 \mathrm{rpm}$. Magnification $=1000 \mathrm{X}$.

Table 2: Bulk density, true density and total porosity of scaffolds.

\begin{tabular}{lcccc}
\hline Scaffold samples & & $\begin{array}{c}\text { Bulk density, } \\
\rho_{\text {scaffold }}\left(\mathrm{g} \mathrm{cm}^{-3}\right)\end{array}$ & $\begin{array}{c}\text { True density, } \\
\rho_{\text {material }}\left(\mathrm{g} \mathrm{cm}^{-3}\right)\end{array}$ & $\begin{array}{c}\text { Total porosity } \\
(\%)\end{array}$ \\
\hline Conventional SCPL & & 0.2286 & 1.2517 & 81.74 \\
Enhanced SCPL & Centrifuge $1500 \mathrm{rpm}$ & 0.1433 & 1.2517 & 88.55 \\
& Centrifuge $2000 \mathrm{rpm}$ & 0.1377 & 1.2517 & 89.00 \\
& Centrifuge 2500 rpm & 0.1200 & 1.2517 & 90.42 \\
& Centrifuge 3000 rpm & 0.1511 & 1.2517 & 87.93 \\
\hline
\end{tabular}

\subsection{Compression Testing}

Mechanical properties of composites scaffold are controlled by several microstructural factors such as the properties of the matrix, the properties and distribution of fillers, interfacial bonding strength and processing methods. ${ }^{2}$ For composite scaffolds, BG particles help to improve the tensile properties of the scaffolds. Figures 4(a and b) show the compressive strength and compressive modulus of scaffold samples at 50\% compressive strain. Compressive strength of scaffolds fabricated by enhanced SCPL method was lower than that of conventional SCPL 
method. Subsequently, compressive modulus of scaffolds obtained also showed the same trend. As centrifugal speed increases from $1500 \mathrm{rpm}$ to $2500 \mathrm{rpm}$, compressive strength shows a decrement values from $0.11 \mathrm{MPa}$ to $0.04 \mathrm{MPa}$. However, the compressive strength decreased to $0.18 \mathrm{Mpa}$ when the centrifugal speed was increased to $3000 \mathrm{rpm}$. Compressive modulus also showed a parallel trend with the compressive strength.

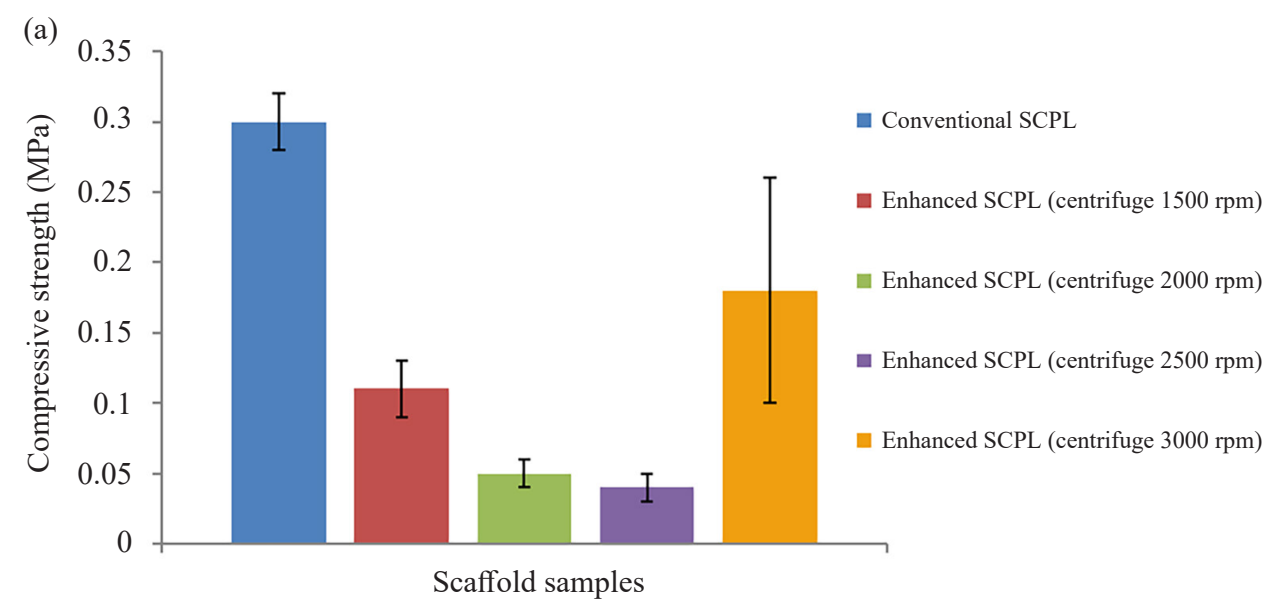

(b)

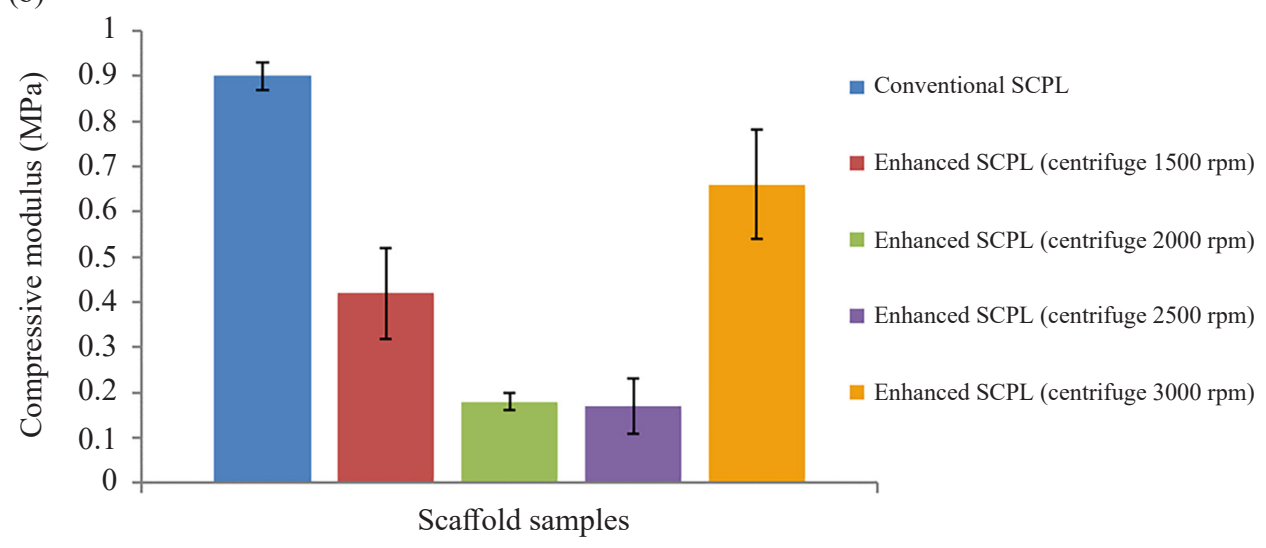

Figure 4: Illustrations of (a) compressive strength, and (b) compressive modulus of scaffold at $50 \%$ compressive strain. 
Compressive strength and modulus were inversely proportional to the total porosity. Loh and choong stated that higher porosity and pore sizes may facilitate nutrient and oxygen delivery or enable more cell ingrowth but the mechanical properties of the scaffolds will be compromised due to the large amount of void volume. ${ }^{9}$ Although enhanced SCPL method shows higher porosity than conventional SCPL method, lower compressive strength of scaffolds was obtained. The presence of more interconnected pores using enhanced SCPL method results in lower compressive strength than conventional SCPL method. Scaffolds with very low compressive strength properties $(<12 \mathrm{MPa})$ were correlated with the natural compressive strength of trabecular bone and can be used for non-load-bearing applications. ${ }^{10}$ These scaffolds were generally very porous ( $80 \%$ or above) which is essential for bone ingrowth. In this study, the values of compressive strength and total porosity fulfill the above requirement.

\section{CONCLUSION}

Bioactive composite scaffold containing PU and 45S5 bioactive glass was fabricated by conventional SCPL method and enhanced SCPL method. The fabricated scaffolds using enhanced SCPL method possess higher porosity than conventional SCPL method. The total porosity of scaffolds fabricated by enhanced SCPL method is ranging approximately from $88 \%$ to $90 \%$, whereas conventional SCPL method is approximately $81 \%$. The pore size of scaffolds fabricated by conventional SCPL and enhanced SCPL methods varied due to the various size and shape of the leaching agent. The overall pore sizes of all fabricated scaffolds were in the range of $96 \mu \mathrm{m}$ to $460 \mu \mathrm{m}$. The scaffolds fabricated by enhanced SCPL method possess high pore interconnectivity as there were more macro-porous structures with micro-pores present in the scaffold walls. This high pore interconnected structure was due to the centrifugal force which enabled the salt particles to be well distributed and densely packed. Scaffolds fabricated by conventional SCPL showed good mechanical properties as compared to enhanced SCPL method. Compressive strength of the scaffolds fabricated by conventional SCPL method was around $0.90 \mathrm{MPa}$ while for the enhanced SCPL method, compressive strength of the scaffolds was in the range of $0.17 \mathrm{MPa}$ to $0.66 \mathrm{MPa}$. Despite having lower compressive strength, scaffold fabricated by SCPL method are considered as mechanically compatible for a low load-bearing application.

\section{ACKNOWLEDGEMENTS}

This work was supported by Ministry of Education Malaysia via Fundamental Research Grant Scheme (FRGS) with grant number 203.PBAHAN.6071323. 


\section{REFERENCES}

1. Stratton, S. et al. (2016). Bioactive materials bioactive polymeric scaffolds for tissue engineering. Bioact. Mater, 1, 93-10, https://doi.org/10.1016/j. bioactmat.2016.11.001.

2. Tetteh, G. et al. (2014). Electrospun polyurethane/hydroxyapatite bioactive scaffolds for bone tissue engineering: The role of solvent and hydroxyapatite particles. J. Mech. Behav. Biomed. Mater., 39, 95-110, https://doi.org/10.1016/j. jmbbm.2014.0 6.019.

3. Sin, D. C. et al. (2010). Polyurethane (PU) scaffolds prepared by solvent casting/ particulate leaching (SCPL) combined with centrifugation. Mater. Sci. Eng. C, 30, 78-85, https://doi.org/10.1016/j.msec.2009.09.002.

4. Turnbull, G., Clarke, J. \& Picard, F. (2018). Bioactive materials 3D bioactive composite scaffolds for bone tissue engineering. Bioact. Mater., 3, 278-314, https://doi.org/10.1016/j.bioactmat.2017.10.00.

5. Zeimaran, E. et al. (2015). Bioactive glass reinforced elastomer composites for skeletal regeneration: A review. Mater. Sci. Eng. C., 53, 175-188, https://oi. org/10.1016/j.msec.2015.04.035.

6. Zhu, N. \& Chen, X. (2013). Advances in biomaterials science and biomedical applications. Saskatoon, Canada: InTech.

7. Bil, M. et al. (2009). Optimization of the structure of polyurethanes for bone tissue engineering applications. Acta Biomater., 6, 2501-2510, https://doi.org/10.1016/j. actbio.2009.08.037.

8. Suhaimin, I. S., Zubir, S. A. \& Abdullah, T. K. (2018). Effect of leaching agent composition on morphology, thermal and mechanical properties of Bioglass ${ }^{\circledR}$ reinforced polyurethane scaffold. Int. J. Curr. Res. Sci. Eng. Technol., 19, 19-27, http://doi.org/10.30967/ijcrset.1.s1.2018.19-27.

9. Loh, Q. L. et al. (2013). Three-dimensional scaffolds for tissue engineering applications: Role of porosity and pore size. Tiss. Eng., 19, 485-502, https://doi. org/10.1089/ten.teb.2012.0437.

10. Pilia, M., Guda, T. \& Appleford, M. (2013). Development of composite scaffolds for load-bearing segmental bone defects. Biomed. Res. Int., 35, 1-15, https://doi. org/10.1155/2013/458253. 